\title{
BOUNDARY CONCENTRATION OF A GAUGED NONLINEAR SCHRÖDINGER EQUATION ON LARGE BALLS
}

\author{
ALESSIO POMPONIO ${ }^{1}$ AND DAVID RUIZ ${ }^{2}$
}

\begin{abstract}
This paper is motivated by a gauged Schrödinger equation in dimension 2 including the so-called Chern-Simons term. The radially symmetric case leads to an elliptic problem with a nonlocal defocusing term, in competition with a local focusing nonlinearity. In this work we pose the equations in a ball under homogeneous Dirichlet boundary conditions. By using singular perturbation arguments we prove existence of solutions for large values of the radius. Those solutions are located close to the boundary and the limit profile is given.
\end{abstract}

\section{INTRODUCTION}

Let us consider a gauged Nonlinear Schrödinger Equation in the plane:

$$
i D_{0} \phi+\left(D_{1} D_{1}+D_{2} D_{2}\right) \phi+|\phi|^{p-1} \phi=0 .
$$

Here $t \in \mathbb{R}, x=\left(x_{1}, x_{2}\right) \in \mathbb{R}^{2}, \phi: \mathbb{R} \times \mathbb{R}^{2} \rightarrow \mathbb{C}$ is the scalar field, $A_{\mu}: \mathbb{R} \times \mathbb{R}^{2} \rightarrow \mathbb{R}$ are the components of the gauge potential and $D_{\mu}=\partial_{\mu}+i A_{\mu}$ is the covariant derivative $(\mu=0,1,2)$.

The natural equations for the gauge potential $A_{\mu}$ are the Maxwell equations. However, the modified gauge field equation proposes to include also the so-called Chern-Simons term, that is,

$$
\partial_{\mu} F^{\mu \nu}+\frac{1}{2} \kappa \epsilon^{\nu \alpha \beta} F_{\alpha \beta}=j^{\nu} \text {, with } F_{\mu \nu}=\partial_{\mu} A_{\nu}-\partial_{\nu} A_{\mu} .
$$

In the above equation, $\kappa$ is a parameter that measures the strength of the ChernSimons term. As usual, $\epsilon^{\nu \alpha \beta}$ is the Levi-Civita tensor, and super-indices are related to the Minkowski metric with signature $(1,-1,-1)$. Finally, $j^{\mu}$ is the conserved matter current,

$$
j^{0}=|\phi|^{2}, j^{i}=2 \operatorname{Im}\left(\bar{\phi} D_{i} \phi\right) .
$$

At low energies, the Maxwell term becomes negligible and can be dropped, giving rise to:

$$
\frac{1}{2} \kappa \epsilon^{\nu \alpha \beta} F_{\alpha \beta}=j^{\nu}
$$

See $[6,7,11,12,13]$ and $[15$, Chapter 1$]$ for the discussion above.

2010 Mathematics Subject Classification. 35J20, 35Q55.

Key words and phrases. Gauged Schrödinger Equations, Lyapunov-Schmidt reduction.

A.P. is supported by M.I.U.R. - P.R.I.N. "Metodi variazionali e topologici nello studio di fenomeni non lineari", by GNAMPA Project "Metodi Variazionali e Problemi Ellittici Non Lineari" and by FRA2011 "Equazioni ellittiche di tipo Born-Infeld". D.R. is supported by the Spanish Ministry of Science and Innovation under Grant MTM2011-26717 and by J. Andalucia (FQM 116). 
For the sake of simplicity, let us normalize so that $\kappa=2$. Equations (1) and (2) lead us to the problem:

$$
\begin{aligned}
& i D_{0} \phi+\left(D_{1} D_{1}+D_{2} D_{2}\right) \phi+|\phi|^{p-1} \phi=0, \\
& \partial_{0} A_{1}-\partial_{1} A_{0}=\operatorname{Im}\left(\bar{\phi} D_{2} \phi\right), \\
& \partial_{0} A_{2}-\partial_{2} A_{0}=-\operatorname{Im}\left(\bar{\phi} D_{1} \phi\right), \\
& \partial_{1} A_{2}-\partial_{2} A_{1}=\frac{1}{2}|\phi|^{2} .
\end{aligned}
$$

As usual in Chern-Simons theory, problem (3) is invariant under gauge transformation,

for any arbitrary $C^{\infty}$ function $\chi$.

$$
\phi \rightarrow \phi e^{i \chi}, \quad A_{\mu} \rightarrow A_{\mu}-\partial_{\mu} \chi
$$

This model was first proposed and studied in [11, 12, 13], and sometimes has received the name of Chern-Simons-Schrödinger equation. The initial value problem, as well as global existence and blow-up, has been addressed in $[4,8,10]$ for the case $p=3$.

The existence of stationary states for (3) and general $p>1$ has been studied recently in $[5,9,14]$. By using the ansatz:

$$
\begin{array}{ll}
\phi(t, x)=u(|x|) e^{i \omega t}, & A_{0}(x)=A_{0}(|x|), \\
A_{1}(t, x)=-\frac{x_{2}}{|x|^{2}} h(|x|), & A_{2}(t, x)=\frac{x_{1}}{|x|^{2}} h(|x|),
\end{array}
$$

and after some manipulations, in [5] it is found that:

(4) $-\Delta u(x)+\left(\omega+\frac{h^{2}(|x|)}{|x|^{2}}+\int_{|x|}^{+\infty} \frac{h(s)}{s} u^{2}(s) d s\right) u(x)=|u(x)|^{p-1} u(x), \quad$ in $\mathbb{R}^{2}$,

where

Here $A_{0}$ takes the form:

$$
h(r)=\frac{1}{2} \int_{0}^{r} s u^{2}(s) d s
$$

$$
A_{0}(r)=\int_{r}^{+\infty} \frac{h(s)}{s} u^{2}(s) d s .
$$

Observe that (4) is a nonlocal equation. Moreover, in [5] it is shown that (4) is indeed the Euler-Lagrange equation of the energy functional:

$$
I: H_{r}^{1}\left(\mathbb{R}^{2}\right) \rightarrow \mathbb{R},
$$

defined as

$$
\begin{aligned}
I(u)= & \frac{1}{2} \int_{\mathbb{R}^{2}}\left(|\nabla u(x)|^{2}+\omega u^{2}(x)\right) d x \\
& +\frac{1}{8} \int_{\mathbb{R}^{2}} \frac{u^{2}(x)}{|x|^{2}}\left(\int_{0}^{r} s u^{2}(s) d s\right)^{2} d x-\frac{1}{p+1} \int_{\mathbb{R}^{2}}|u(x)|^{p+1} d x .
\end{aligned}
$$

Here $H_{r}^{1}\left(\mathbb{R}^{2}\right)$ is the Sobolev space of radially symmetric functions. In $[5,9,14]$ critical points of $I$ are found by using variational methods. It is shown that the value $p=3$ is critical for this problem. Indeed, for $p>3$ the energy functional is unbounded from below and satisfies a mountain-pass geometry. Even if the (PS) property is not known to be satisfied, positive solutions are found in [5].

Instead, for $p \in(1,3)$ the global behavior of $I$ is different and depends on the parameter $\omega$. In [14] we show that $I$ is bounded from below if and only if $\omega \geqslant \omega_{0}$, where the threshold value $\omega_{0}$ is explicit, namely:

$$
\omega_{0}=\frac{3-p}{3+p} 3^{\frac{p-1}{2(3-p)}} 2^{\frac{2}{3-p}}\left(\frac{m^{2}(3+p)}{p-1}\right)^{-\frac{p-1}{2(3-p)}} .
$$


Here $m=\int_{-\infty}^{+\infty} w_{1}^{2}(r) d r$, and $w_{1}$ is the unique positive even solution of the problem $-w^{\prime \prime}+w=w^{p}$ in $\mathbb{R}$, i.e.,

$$
w_{1}(r)=\left(\frac{2}{p+1} \cosh ^{2}\left(\frac{p-1}{2} r\right)\right)^{\frac{1}{1-p}} .
$$

In this paper we study positive solutions for the Dirichlet problem posed on a ball $B(0, R)$, that is:

(6)

$$
\begin{cases}-\Delta u(x)+\left(\omega+\frac{h^{2}(|x|)}{|x|^{2}}+\int_{|x|}^{R} \frac{h(s)}{s} u^{2}(s) d s\right) u(x)=u^{p}(x), & x \in B(0, R), \\ u(x)=0, & x \in \partial B(0, R),\end{cases}
$$

where, as above,

$$
h(r)=\frac{1}{2} \int_{0}^{r} s u^{2}(s) d s .
$$

Let us denote by $H_{R}=H_{0, r}^{1}(B(0, R))$ the Sobolev Space of radially symmetric functions. Again, (6) is the Euler-Lagrange equation of the functional $I_{R}: H_{R} \rightarrow$ $\mathbb{R}, I_{R}=\left.I\right|_{H_{R}}$.

A first motivation for this study comes from the proofs of [14]. It is not difficult to show that $I_{R}$ is coercive for any $R$ fixed. So, there exists a minimizer $u_{R}$ of $I_{R}$. In order to prove boundedness of $u_{R}$ when $R \rightarrow+\infty$, the problem is the possible loss of mass at infinity. In [14] we prove that, if unbounded, the sequence $u_{R}$ actually behaves as a soliton, when $u_{R}$ is interpreted as a function of a single real variable. Then, the energy functional $I$ admits a natural approximation through a convenient limit functional $J$, see Section 2 for details. Moreover, the solutions of that limit functional, and their energy, can be found explicitly, and this leads us to a contradiction if $\omega \geqslant \omega_{0}$.

In this paper we show that this kind of solutions actually exists for $\omega<\omega_{0}$. Moreover, their energy tends to $-\infty$ as $R \rightarrow+\infty$, and they seem to be local minima of $I$. Therefore, they could correspond to global minima of $I$, which is an interesting phenomenon: the minima of the energy tend to aggregate near the boundary of the domain.

A second motivation comes from reference [3]. Observe that for any $u$, the function:

$$
\frac{h^{2}(|x|)}{|x|^{2}}+\int_{|x|}^{R} \frac{h(s)}{s} u^{2}(s) d s,
$$

is decreasing in $|x|$. Therefore, a solution like in [3] could exist. However here the problem is completely different, since $h$ itself depends on $u$ in a nonlocal fashion. Indeed, natural restrictions on $\omega$ and $p$ appear, as commented above.

The main result of this paper is the following:

Theorem 1.1. Assume $p \in(1,3), \omega \in\left(0, \omega_{0}\right)$. Then, for $R$ large enough, problem (6) admits a positive solution $\tilde{u}_{R} \sim U(r-\rho)$, with

$$
U(r)=k_{2}^{\frac{1}{p-1}} w_{1}\left(\sqrt{k_{2}} r\right) .
$$

Here $w_{1}$ is given by (5) and $k_{2}$ is the largest positive root of the equation:

$$
k=\omega+\frac{1}{4} m^{2} k^{\frac{5-p}{p-1}}, k>0 .
$$

Finally,

$$
\rho \sim R-\frac{1}{2 \sqrt{k_{2}}} \log R
$$


As commented above, the assumption $\omega \in\left(0, \omega_{0}\right)$ is natural for this result. Moreover, observe that $\omega_{0}$ is well defined only for $p \in(1,3)$, and it tends to 0 as $p$ tends to 3 .

The proof uses a singular perturbation method based on a Lyapunov-Schmidt reduction, which has become a well-known technique in this kind of problems. However, there are some new aspects on the problem under consideration. First, our problem involves a nonlocal term which makes the argument of the proof more complicated. We point out that it is not just a technical difficulty, since the existence result depends heavily on the nonlocal term. Indeed, those solutions do not exist for the autonomous Nonlinear Schrödinger Equation, and are a special feature of the model studied in this paper.

Moreover, the limit functional is different from the usual one in Nonlinear Schrödinger Equations and contains also a nonlocal term. As a consequence, we also need to prove the non-degeneracy of the limit solution, which in this case corresponds to a global minimum of the limit functional.

The rest of the paper is organized as follows. Section 2 is devoted to some preliminary results. The limit functional and its properties are exposed here. Moreover, we prove that the limit solution is non-degenerate. The last two sections are devoted to the proof of Theorem 1.1. Section 3 is devoted to solve the auxiliary equation in the Lyapunov-Schmidt reduction, whereas in Section 4 we prove the existence of a minimum for the reduced functional.

Acknowledgement. This work has been partially carried out during a stay of A.P. in Granada. He would like to express his deep gratitude to the Departamento de Análisis Matemático for the support and warm hospitality.

\section{PRELIMINARIES}

Let us first fix some notations. We denote by $H_{R}=H_{0, r}^{1}(B(0, R))$ the Sobolev space of radially symmetric functions. We use $\langle\cdot\rangle,\|\cdot\|$ to denote its usual scalar product and norm, whereas other norms, like Lebesgue norms in $B(0, R) \subset \mathbb{R}^{2}$, will be indicated with a subscript. Moreover, $\langle\cdot\rangle_{H^{1}(a, b)},\|\cdot\|_{H^{1}(a, b)}$ are used to indicate the scalar product and norm of the Sobolev space of dimension 1. If nothing is specified, strong and weak convergence of sequences of functions are assumed in the space $H_{R}$.

In our estimates, we will frequently denote by $C>0, c>0$ fixed constants, that may change from line to line, but are always independent of the variable under consideration. We also use the notations $O(1), o(1), O(\varepsilon), o(\varepsilon)$ to describe the asymptotic behaviors of quantities in a standard way. Finally the letter $x$ indicates a two-dimensional variable, and $r, s, t$ denote one-dimensional variables.

Let us start with the following proposition:

Proposition 2.1. $I_{R}$ is a $C^{1}$ functional, and its critical points correspond to classical solutions of (6). 
This was proved in [5] for the problem set in $\mathbb{R}^{2}$; the same arguments apply to our case. Moreover, the derivative of $I_{R}$ is:

$$
\begin{aligned}
\frac{I_{R}^{\prime}(z)[u]}{2 \pi}= & \int_{0}^{R}\left(z^{\prime} u^{\prime}+\omega z u-|z|^{p-2} z u\right) r d r \\
& +\frac{1}{4} \int_{0}^{R} \frac{z(r) u(r)}{r}\left(\int_{0}^{r} z^{2}(s) s d s\right)^{2} d r \\
& +\frac{1}{2} \int_{0}^{R} \frac{z^{2}(r)}{r}\left(\int_{0}^{r} z^{2}(t) t d t\right)\left(\int_{0}^{r} z(s) u(s) s d s\right) d r .
\end{aligned}
$$

If in the last term we use Fubini Theorem in the variables $r, s$, and after that we interchange the name of the variables, we obtain:

$$
\begin{aligned}
\frac{I_{R}^{\prime}(z)[u]}{2 \pi}= & \int_{0}^{R}\left(z^{\prime} u^{\prime}+\omega z u-|z|^{p-2} z u\right) r d r \\
& +\frac{1}{4} \int_{0}^{R} \frac{z(r) u(r)}{r}\left(\int_{0}^{r} z^{2}(s) s d s\right)^{2} d r \\
& +\frac{1}{2} \int_{0}^{R} z(r) u(r) r\left(\int_{r}^{R} \frac{z^{2}(s)}{s}\left(\int_{0}^{s} z^{2}(t) t d t\right) d s\right) d r .
\end{aligned}
$$

In this paper we will make use of both expressions for $I_{R}^{\prime}$, depending on the case.

Our proof of Theorem 1.1 uses a Lyapunov-Schmidt reduction to prove existence of a critical point for $I_{R}$. In order to do that, some knowledge of the limit functional is needed. First of all, let us give a heuristic derivation of this energy functional. Consider $u(r)$ a fixed function, and define $u_{\rho}(r)=u(r-\rho)$. Let us now estimate $I\left(u_{\rho}\right)$ as $\rho \rightarrow+\infty$; after the change of variables $r \rightarrow r+\rho$, we obtain:

$$
\begin{aligned}
\frac{I\left(u_{\rho}\right)}{2 \pi}= & \frac{1}{2} \int_{-\rho}^{+\infty}\left(\left|u^{\prime}\right|^{2}+\omega u^{2}\right)(r+\rho) d r \\
& +\frac{1}{8} \int_{-\rho}^{\infty} \frac{u^{2}(r)}{r+\rho}\left(\int_{-\rho}^{r}(s+\rho) u^{2}(s) d s\right)^{2} d r-\frac{1}{p+1} \int_{-\rho}^{\infty}|u|^{p+1}(r+\rho) d r .
\end{aligned}
$$

We estimate the above expression by simply replacing the expressions $(r+\rho)$, $(s+\rho)$ with the constant $\rho$ :

$$
\begin{aligned}
& \sim \rho\left[\frac{1}{2} \int_{-\infty}^{+\infty}\left(|u|^{2}+\omega u^{2}\right) d r+\frac{1}{8} \int_{-\infty}^{+\infty} u^{2}(r)\left(\int_{-\infty}^{r} u^{2}(s) d s\right)^{2} d r-\frac{1}{p+1} \int_{-\infty}^{+\infty}|u|^{p+1} d r\right] \\
& \quad=\rho\left[\frac{1}{2} \int_{-\infty}^{+\infty}\left(|u|^{\prime 2}+\omega u^{2}\right) d r+\frac{1}{24}\left(\int_{-\infty}^{+\infty} u^{2} d r\right)^{3}-\frac{1}{p+1} \int_{-\infty}^{+\infty}|u|^{p+1} d r\right] .
\end{aligned}
$$

Therefore, it is natural to consider the limit functional $J: H^{1}(\mathbb{R}) \rightarrow \mathbb{R}$,

(9) $J(u)=\frac{1}{2} \int_{-\infty}^{+\infty}\left(\left|u^{\prime}\right|^{2}+\omega u^{2}\right) d r+\frac{1}{24}\left(\int_{-\infty}^{+\infty} u^{2} d r\right)^{3}-\frac{1}{p+1} \int_{-\infty}^{+\infty}|u|^{p+1} d r$.

Clearly, the Euler-Lagrange equation of (9) is the following limit problem:

$$
-u^{\prime \prime}+\omega u+\frac{1}{4}\left(\int_{-\infty}^{+\infty} u^{2}(s) d s\right)^{2} u=|u|^{p-1} u, \quad \text { in } \mathbb{R} .
$$

Before going on with the study of (10), we need some well-known facts concerning the problem

$$
-w^{\prime \prime}+k w=w^{p} \quad \text { in } \mathbb{R}, k>0 .
$$


We denote by $w_{k} \in H^{1}(\mathbb{R})(k>0)$ the unique positive even solution of (11). Observe that equation (11) is integrable and the Hamiltonian of $w_{k}$ is equal to 0 , that is,

$$
-\frac{1}{2}\left|w_{k}^{\prime}(r)\right|^{2}+\frac{k}{2} w_{k}^{2}(r)-\frac{1}{p+1} w_{k}^{p+1}(r)=0, \text { for all } r \in \mathbb{R} .
$$

It is also known that any positive solution of (11) is of the form $u(r)=w_{k}(r-\xi)$, for some $\xi \in \mathbb{R}$. Moreover,

$$
w_{k}(r)=k^{\frac{1}{p-1}} w_{1}(\sqrt{k} r), \quad \text { and } \quad w_{1}(r)=\left(\frac{2}{p+1} \cosh ^{2}\left(\frac{p-1}{2} r\right)\right)^{\frac{1}{1-p}} .
$$

Notice that $w_{k}$ and $w_{k}^{\prime}$ decay exponentially to zero at infinity.

In what follows we define

$$
m=\int_{-\infty}^{+\infty} w_{1}^{2} d r
$$

The following relations are also well known, and can be deduced from (12):

$$
\int_{-\infty}^{+\infty}\left|w_{1}^{\prime}\right|^{2} d r=\frac{p-1}{p+3} m, \quad \int_{-\infty}^{+\infty} w_{1}^{p+1} d r=\frac{2(p+1)}{p+3} m .
$$

Let us now come back to equation (10). Consider $u$ a positive solution of (10), and define $k=\omega+\frac{1}{4}\left(\int_{-\infty}^{+\infty} u^{2} d r\right)^{2}$. Then, $u$ is a solution of $-u^{\prime \prime}+k u=u^{p}$, so $u(r)=w_{k}(r-\xi)$ for some $\xi \in \mathbb{R}$. By using (13), we obtain:

$$
k=\omega+\frac{1}{4}\left(\int_{-\infty}^{+\infty} w_{k}^{2}(r) d r\right)^{2}=\omega+\frac{1}{4} k^{\frac{4}{p-1}}\left(\int_{-\infty}^{+\infty} w_{1}^{2}(\sqrt{k} r) d r\right)^{2} .
$$

A change of variables leads us to the identity:

$$
k=\omega+\frac{1}{4} m^{2} k^{\frac{5-p}{p-1}}, k>0 .
$$

Therefore, the existence of solutions for (10) reduces to the existence of solutions of the algebraic equation (16). Moreover, we are also interested in the energy of those solutions, as we shall see in Section 4 . Those questions have been treated in [14, Section 3], where the following results were obtained:

Proposition 2.2. Assume $p \in(1,3)$ and take $m$ as in (14). Define:

$$
\begin{aligned}
& \omega_{0}=\frac{3-p}{3+p} 3^{\frac{p-1}{2(3-p)}} 2^{\frac{2}{3-p}}\left(\frac{m^{2}(3+p)}{p-1}\right)^{-\frac{p-1}{2(3-p)}}, \\
& \omega_{1}=\left(\frac{(5-p) m^{2}}{4(p-1)}\right)^{-\frac{p-1}{2(3-p)}}-\frac{m^{2}}{4}\left(\frac{(5-p) m^{2}}{4(p-1)}\right)^{-\frac{(5-p)}{2(3-p)}} .
\end{aligned}
$$

The following holds:

(1) $0<\omega_{0}<\omega_{1}$;

(2) if $\omega>\omega_{1}$, equation (16) has no solution and there is no nontrivial solution of (10);

(3) if $\omega=\omega_{1}$, equation (16) has only one solution $k_{0}$ and $w_{k_{0}}(r)$ is the only positive solution of (10) (apart from translations);

(4) if $\omega \in\left(0, \omega_{1}\right)$, equation (16) has two solutions $k_{1}(\omega)<k_{2}(\omega)$ and $w_{k_{1}}(r), w_{k_{2}}(r)$ are the only two positive solutions of (10) (apart from translations);

(5) for any $\omega \in\left(0, \omega_{1}\right), J\left(w_{k_{1}}\right)>0$. 
(6) $J\left(w_{k_{2}}\right)<0$ if and only if $\omega \in\left(0, \omega_{0}\right)$. In such case, $w_{k_{2}}$ is a global minimizer for $J$.

Remark 2.3. In general, we cannot obtain a more explicit expression of $m$ depending on $p$, but it can be easily approximated by using some software. In Figure 1 the maps $\omega_{0}(p)$ and $\omega_{1}(p)$ have been plotted.

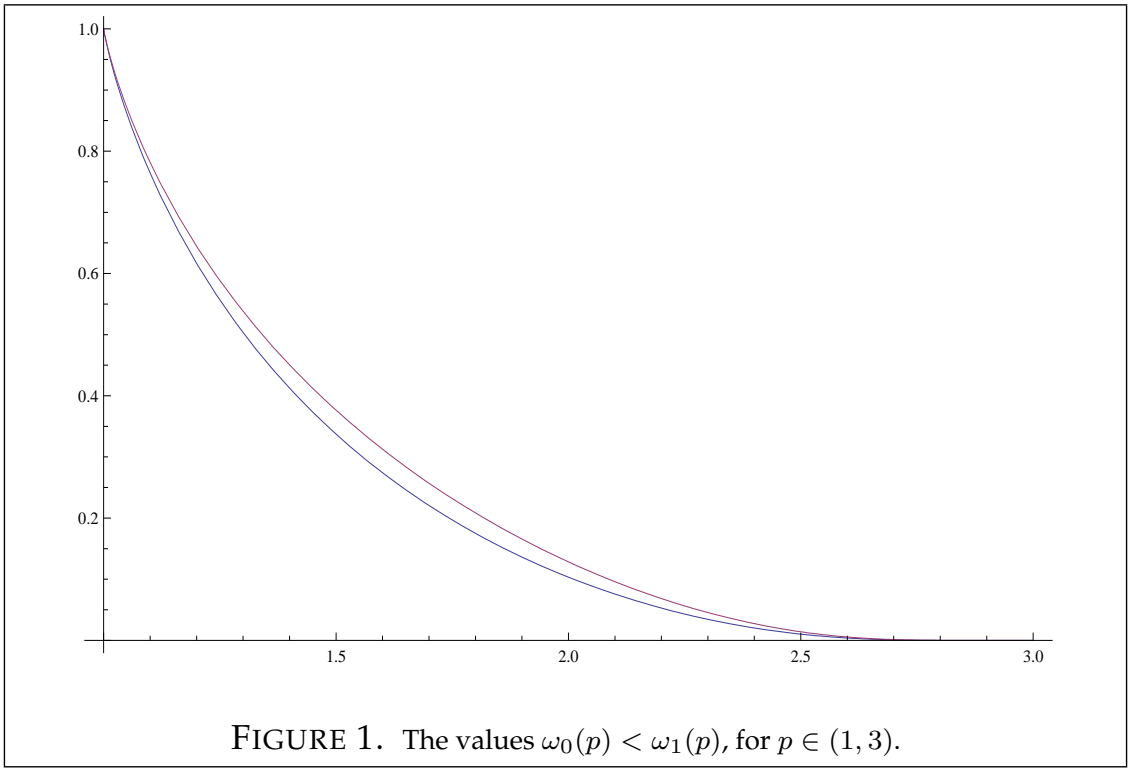

For some specific values of $p, m$ can be explicitly computed, and hence $\omega_{0}$ and $\omega_{1}$. For instance, if $p=2, m=6, \omega_{1}=\frac{2}{9 \sqrt{3}}$ and $\omega_{0}=\frac{2}{5 \sqrt{15}}$.

As commented in the introduction, in Section 3 we will use the above solutions as a limit profile of solutions of (6) via a Lyapunov-Schmidt reduction. On that purpose, the non-degeneracy of the limit solutions is required. We say that a solution $u$ of (10) is degenerate if the problem:

$$
\begin{aligned}
-\phi^{\prime \prime}+\omega \phi+\frac{1}{4} & \left(\int_{-\infty}^{+\infty} u^{2}(s) d s\right)^{2} \phi \\
& +\left(\int_{-\infty}^{+\infty} u^{2}(s) d s\right)\left(\int_{-\infty}^{+\infty} u(s) \phi(s) d s\right) u-p u^{p-1} \phi=0
\end{aligned}
$$

has a solution $\phi \in H^{1}(\mathbb{R})$ different from $c u^{\prime}(r), c \in \mathbb{R}$.

Next proposition is devoted to this question.

Proposition 2.4. Let $\omega_{1}, k_{0}, k_{1}, k_{2}$ as in Proposition 2.2. Then:

(1) If $\omega=\omega_{1}, w_{k_{0}}$ is a degenerate solution of (10).

(2) If $\omega \in\left(0, \omega_{1}\right)$, both $w_{k_{1}}$ and $w_{k_{2}}$ are non-degenerate solutions of (10).

Proof. Take $w_{k}$ a solution of (10), and $\phi$ a solution of (17). Define

$$
\alpha=\left(\int_{-\infty}^{+\infty} w_{k}^{2}(s) d s\right)\left(\int_{-\infty}^{+\infty} w_{k}(s) \phi(s) d s\right) \in \mathbb{R} .
$$

Then, $\phi$ is a solution of the problem:

$$
-\phi^{\prime \prime}+\omega \phi+\frac{1}{4}\left(\int_{-\infty}^{+\infty} w_{k}^{2}(s) d s\right)^{2} \phi-p w_{k}^{p-1} \phi=-\alpha w_{k} .
$$


Write $\phi(r)=\psi(r) w_{k}^{\prime}(r)$ : then,

$$
\psi^{\prime \prime}(r)=-2 \psi^{\prime}(r) \frac{w_{k}^{\prime \prime}(r)}{w_{k}^{\prime}(r)}+\alpha \frac{w_{k}(r)}{w_{k}^{\prime}(r)} .
$$

This is a linear equation of first order in $\psi^{\prime}(r)$, which can be solved by variation of constants:

$$
\psi^{\prime}(r)=\frac{C}{\left(w_{k}^{\prime}(r)\right)^{2}}+\frac{\alpha}{2} \frac{w_{k}^{2}(r)}{\left(w_{k}^{\prime}(r)\right)^{2}}, C \in \mathbb{R} .
$$

Observe that if $C \neq 0$, then $\phi(r)=\psi(r) w_{k}^{\prime}(r)$ tends to $+\infty$ at infinity, which is not possible if $\phi \in H^{1}(\mathbb{R})$. So, $C$ must be equal to 0 , and we are led to:

$$
\psi^{\prime}(r)=\frac{\alpha}{2} \frac{w_{k}^{2}(r)}{\left(w_{k}^{\prime}(r)\right)^{2}} .
$$

By using (12), the above equation can be explicitly integrated:

$$
\psi(r)=\frac{\alpha}{2 k}\left(\frac{2}{(p-1)} \frac{w_{k}(r)}{w_{k}^{\prime}(r)}+r\right)+\beta, \quad \text { with } \beta \in \mathbb{R} .
$$

Therefore,

$$
\phi(r)=\frac{\alpha}{2 k}\left(\frac{2}{p-1} w_{k}(r)+r w_{k}^{\prime}(r)\right)+\beta w_{k}^{\prime}(r) .
$$

Clearly, $w_{k}$ is degenerate if and only if $\alpha$ can take values different from zero. Take $\beta=0$, and recall now the definition of $\alpha$ :

$$
\alpha=\frac{\alpha}{2 k}\left(\int_{-\infty}^{+\infty} w_{k}^{2}(s) d s\right)\left(\int_{-\infty}^{+\infty} \frac{2}{p-1} w_{k}^{2}(s)+s w_{k}^{\prime}(s) w_{k}(s) d s\right) .
$$

By integration by parts,

$$
\int_{-\infty}^{+\infty} s w_{k}^{\prime}(s) w_{k}(s) d s=-\frac{1}{2} \int_{-\infty}^{+\infty} w_{k}^{2}(s) d s=-\frac{1}{2} k^{\frac{5-p}{2(p-1)}} m .
$$

Then, either $\alpha=0$ or

$$
1=m^{2} k^{\frac{6-2 p}{p-1}} \frac{5-p}{4(p-1)} .
$$

This, together with (16), implies that $\omega=\omega_{1}$ and $k=k_{0}$.

Observe that, due to the nonlocal character of our problem, the linearized problem (17) does not have the form of a Schrödinger operator. Then, some more work is needed in order to accomplish a Lyapunov-Schmidt reduction. Let us define the following operators:

$$
\begin{aligned}
T[\phi] & =-\phi^{\prime \prime}+\left[\omega+\frac{1}{4}\left(\int_{-\infty}^{+\infty} u^{2}(s) d s\right)^{2}-p u^{p-1}\right] \phi, \\
K[\phi] & =\left(\int_{-\infty}^{+\infty} u^{2}(s) d s\right)\left(\int_{-\infty}^{+\infty} u(s) \phi(s) d s\right) u, \\
L[\phi] & =T[\phi]+K[\phi] .
\end{aligned}
$$

Observe that $L, T, K: H^{1}(\mathbb{R}) \cap\left\{u^{\prime}\right\}^{\perp} \rightarrow H^{-1}(\mathbb{R}) \cap\left\{u^{\prime}\right\}^{\perp}$. Of course, here orthogonality is understood for the scalar product $\langle\cdot\rangle_{H^{1}(\mathbb{R})}$.

Corollary 2.5. Assume $\omega \in\left(0, \omega_{1}\right)$. Then the operator $L$ defined above is a bijection for $u=w_{k_{1}}$ or $u=w_{k_{2}}$. Moreover, if $\omega<\omega_{0}$ and $u=w_{k_{2}}$, there exists $c>0$ so that:

$$
\langle L[\phi], \phi\rangle_{H^{1}(\mathbb{R})} \geqslant c\|\phi\|_{H^{1}(\mathbb{R})}^{2} \text { for any } \phi \in H^{1}(\mathbb{R}) \cap\left\{u^{\prime}\right\}^{\perp} .
$$


Proof. It is well-known that $T$ is a bijection. Since the image of $K$ is of dimension $1, K$ is obviously a compact operator. Moreover, by Proposition 2.4, $L$ is injective. By Fredholm alternative, $L$ is a bijection.

Moreover, by Proposition 2.2, we know that $w_{k_{2}}$ is a minimizer of $J$ with nondegenerate second derivative. From this, (18) follows.

\section{THE AUXILIARY EQUATION}

In this section we begin the proof of Theorem 1.1, which uses a singular perturbation argument based on a Lyapunov-Schmidt reduction. So, let us assume $p \in(1,3)$ and $\omega \in\left(0, \omega_{0}\right)$ throughout the rest of the paper.

The construction of our approximating solution is largely inspired by [3]. We set $U=w_{k_{2}}$, where $k_{2}$ is the second root of equation (16) (see Proposition 2.2). We will denote $U_{\rho}=U(\cdot-\rho)$, and $V_{\rho}=U_{\rho}(R) e^{\sqrt{k_{2}}(\cdot-R)}$. For later use, observe that $U_{\rho}$ solves the equation

$$
-U_{\rho}^{\prime \prime}+\omega U_{\rho}+\frac{1}{4}\left(\int_{-\infty}^{+\infty} U^{2}(s) d s\right)^{2} U_{\rho}=U_{\rho}^{p}, \quad \text { in } \mathbb{R},
$$

whereas $V_{\rho}$ satisfies

$$
-V_{\rho}^{\prime \prime}+\omega V_{\rho}+\frac{1}{4}\left(\int_{-\infty}^{+\infty} U^{2}(s) d s\right)^{2} V_{\rho}=0, \quad \text { in } \mathbb{R} .
$$

We define our approximating solution as $z_{\rho}=\phi_{R}\left(U_{\rho}-V_{\rho}\right) \in H_{R}$, where $\phi_{R}$ : $[0,+\infty) \rightarrow[0,1]$ is a smooth function such that

$$
\phi_{R}(r)=\left\{\begin{array}{ll}
0 & \text { if } r \in(0, R / 4), \\
1 & \text { if } r \in(R / 2, R),
\end{array} \quad\left|\phi_{R}^{\prime}(r)\right| \leqslant \frac{C}{R} .\right.
$$

Let us define the interval

$$
\mathcal{I}_{R}=\left[R-\frac{\beta}{2 \sqrt{k_{2}}} \log R, R-\frac{\alpha}{2 \sqrt{k_{2}}} \log R\right],
$$

where $\alpha, \beta$ are fixed constants satisfying:

$$
\max \left\{\frac{1}{2}, \frac{1}{p}\right\}<\alpha<1,1<\beta<\min \{2, p\} \alpha .
$$

We are interested in finding critical points for $I$ near the manifold of approximating solutions:

$$
Z=\left\{z_{\rho}, \rho \in \mathcal{I}_{R}\right\}
$$

For any $z_{\rho} \in Z$, consider $T_{z_{\rho}} Z$ the tangent space, spanned by the function:

$$
\dot{z}_{\rho}:=\frac{\partial z_{\rho}}{\partial \rho}=\phi_{R}\left(-U_{\rho}^{\prime}+U_{\rho}^{\prime}(R) e^{\sqrt{k_{2}}(\cdot-R)}\right) .
$$

Take also $W=W_{z_{\rho}}=T_{z_{\rho}} Z^{\perp}$, and let $P, Q$ denote the orthogonal projections onto the spaces $W$ and $T_{z_{\rho}} Z$, respectively. We then decompose the equation:

$$
I_{R}^{\prime}\left(z_{\rho}+w\right)=0 \Leftrightarrow \begin{cases}P I_{R}^{\prime}\left(z_{\rho}+w\right)=0 & \text { (auxiliary equation), } \\ Q I_{R}^{\prime}\left(z_{\rho}+w\right)=0 & \text { (bifurcation equation). }\end{cases}
$$

In this section we use the Banach contraction principle to find a solution $w_{\rho}$ of the auxiliary equation for any $\rho \in \mathcal{I}_{R}$. In order to do that, some estimates are in order.

Our first result is an estimate on $I_{R}^{\prime}\left(z_{\rho}\right)$, which measures in which sense $z_{\rho}$ is an approximating solution. 
Lemma 3.1. For any $\rho \in \mathcal{I}_{R}$, we have that

$$
\left\|I_{R}^{\prime}\left(z_{\rho}\right)\right\|= \begin{cases}O\left(\sqrt{\rho}|R-\rho| e^{-2 \sqrt{k_{2}}(R-\rho)}\right), & \text { if } p \geqslant 2, \\ O\left(\sqrt{\rho} e^{-p \sqrt{k_{2}}(R-\rho)}\right), & \text { if } p<2 .\end{cases}
$$

Proof. Let us recall that, by (8),

$$
\begin{aligned}
\frac{I_{R}^{\prime}\left(z_{\rho}\right)[u]}{2 \pi}= & \int_{0}^{R}\left(z_{\rho}^{\prime} u^{\prime}+\omega z_{\rho} u\right) r d r+\int_{0}^{R} \frac{z_{\rho}(r) u(r)}{r}\left(\int_{0}^{r} \frac{s}{2} z_{\rho}^{2}(s) d s\right)^{2} d r \\
& +\int_{0}^{R} z_{\rho}(r) u(r) r\left(\int_{r}^{R} \frac{z_{\rho}^{2}(s)}{s}\left(\int_{0}^{s} \frac{t}{2} z_{\rho}^{2}(t) d t\right) d s\right) d r-\int_{0}^{R} z_{\rho}^{p} u r d r .
\end{aligned}
$$

Let us evaluate all these integrals in $[0, R / 2]$. We have

$$
\left|\int_{0}^{\frac{R}{2}} z_{\rho}^{\prime} u^{\prime} r d r\right| \leqslant C R^{\frac{1}{2}}\left(\int_{\frac{R}{4}}^{\frac{R}{2}} e^{2 \sqrt{k_{2}}(r-\rho)} d r\right)^{\frac{1}{2}}\|u\| \leqslant C R^{\frac{1}{2}} e^{-\sqrt{k_{2}} \frac{R}{4}}\|u\| .
$$

Analogously,

$$
\left|\int_{0}^{\frac{R}{2}} \omega z_{\rho} u r d r\right| \leqslant C R^{\frac{1}{2}} e^{-\sqrt{k_{2}} \frac{R}{4}}\|u\|
$$

Moreover

$$
\begin{aligned}
\left|\int_{0}^{\frac{R}{2}} \frac{z_{\rho}(r) u(r)}{r}\left(\int_{0}^{r} \frac{s}{2} z_{\rho}^{2}(s) d s\right)^{2} d r\right| & \leqslant C\left(\int_{\frac{R}{4}}^{\frac{R}{2}} R e^{2 \sqrt{k_{2}}(s-\rho)} d s\right)^{2} \int_{\frac{R}{4}}^{\frac{R}{2}}\left|\frac{z_{\rho}(r) u(r)}{r}\right| d r \\
& \leqslant C R^{2} e^{-\sqrt{k_{2}} \frac{R}{2}}\left(\int_{\frac{R}{4}}^{\frac{R}{2}} \frac{z_{\rho}^{2}(r)}{r^{3}} d r\right)^{\frac{1}{2}}\|u\| \\
& \leqslant C R^{\frac{1}{2}} e^{-\sqrt{k_{2}} \frac{3 R}{4}}\|u\| .
\end{aligned}
$$

We can also estimate

$$
\begin{aligned}
\left|\int_{0}^{\frac{R}{2}} z_{\rho}(r) u(r) r\left(\int_{r}^{R} \frac{z_{\rho}^{2}(s)}{s}\left(\int_{0}^{s} \frac{t}{2} z_{\rho}^{2}(t) d t\right) d s\right) d r\right| & \leqslant C\left(\int_{\frac{R}{4}}^{R} z_{\rho}^{2}(s) d s\right)^{2} \int_{\frac{R}{4}}^{\frac{R}{2}}\left|z_{\rho}(r) u(r) r\right| d r \\
& \leqslant C R e^{-\sqrt{k_{2}} \frac{R}{4}}\|u\| .
\end{aligned}
$$

Finally

$$
\left|\int_{0}^{\frac{R}{2}} z_{\rho}^{p} u r d r\right| \leqslant C R^{\frac{p}{p+1}} e^{-p \sqrt{k_{2}} \frac{R}{4}}\|u\|
$$

Therefore

$$
\begin{aligned}
\frac{I_{R}^{\prime}\left(z_{\rho}\right)[u]}{2 \pi}= & \int_{\frac{R}{2}}^{R}\left(z_{\rho}^{\prime} u^{\prime}+\omega z_{\rho} u\right) r d r+\int_{\frac{R}{2}}^{R} \frac{z_{\rho}(r) u(r)}{r}\left(\int_{0}^{r} \frac{s}{2} z_{\rho}^{2}(s) d s\right)^{2} d r \\
& +\int_{\frac{R}{2}}^{R} z_{\rho}(r) u(r) r\left(\int_{r}^{R} \frac{z_{\rho}^{2}(s)}{s}\left(\int_{0}^{s} \frac{t}{2} z_{\rho}^{2}(t) d t\right) d s\right) d r-\int_{\frac{R}{2}}^{R} z_{\rho}^{p} u r d r \\
& +o\left(e^{-\sqrt{k_{2}} \frac{R}{5}}\right)\|u\| .
\end{aligned}
$$


Let us prove that

$$
\begin{aligned}
\frac{I_{R}^{\prime}\left(z_{\rho}\right)[u]}{2 \pi}= & \sqrt{\rho}\left[\int_{\frac{R}{2}}^{R}\left(z_{\rho}^{\prime} u^{\prime}+\omega z_{\rho} u\right) \sqrt{r} d r+\frac{1}{4} \int_{\frac{R}{2}}^{R} z_{\rho}(r) u(r) \sqrt{r}\left(\int_{\frac{R}{2}}^{r} z_{\rho}^{2}(s) d s\right)^{2} d r\right. \\
& \left.+\frac{1}{2} \int_{\frac{R}{2}}^{R} z_{\rho}(r) u(r) \sqrt{r}\left(\int_{r}^{R} z_{\rho}^{2}(s)\left(\int_{\frac{R}{2}}^{s} z_{\rho}^{2}(t) d t\right) d s\right) d r-\int_{\frac{R}{2}}^{R} z_{\rho}^{p} u \sqrt{r} d r\right] \\
& +O\left(R^{-1 / 2}\right)\|u\| .
\end{aligned}
$$

Indeed we have

$$
\begin{aligned}
\left|\int_{\frac{R}{2}}^{R} z_{\rho}^{\prime} u^{\prime} r d r-\int_{\frac{R}{2}}^{R} z_{\rho}^{\prime} u^{\prime} \sqrt{\rho} \sqrt{r} d r\right| & \leqslant\left(\int_{\frac{R}{2}}^{R}\left|z_{\rho}^{\prime}\right|^{2}|\sqrt{r}-\sqrt{\rho}|^{2} d r\right)^{\frac{1}{2}}\|u\| \\
& =\left(\int_{\frac{R}{2}}^{R}\left|z_{\rho}^{\prime}\right|^{2}\left|\frac{r-\rho}{\sqrt{r}+\sqrt{\rho}}\right|^{2} d r\right)^{\frac{1}{2}}\|u\| \leqslant \frac{C}{\sqrt{R}}\|u\| .
\end{aligned}
$$

Therefore

$$
\int_{\frac{R}{2}}^{R} z_{\rho}^{\prime} u^{\prime} r d r=\sqrt{\rho} \int_{\frac{R}{2}}^{R} z_{\rho}^{\prime} u^{\prime} \sqrt{r} d r+O\left(R^{-1 / 2}\right)\|u\| .
$$

Analogously, we get

$$
\begin{aligned}
& \int_{\frac{R}{2}}^{R} z_{\rho} u r d r=\sqrt{\rho} \int_{\frac{R}{2}}^{R} z_{\rho} u \sqrt{r} d r+O\left(R^{-1 / 2}\right)\|u\|, \\
& \int_{\frac{R}{2}}^{R} z_{\rho}^{p} u r d r=\sqrt{\rho} \int_{\frac{R}{2}}^{R} z_{\rho}^{p} u^{\prime} \sqrt{r} d r+O\left(R^{-1 / 2}\right)\|u\| .
\end{aligned}
$$

Let us study the nonlocal terms;

$$
\begin{aligned}
\int_{\frac{R}{2}}^{R} \frac{z_{\rho}(r) u(r)}{r}\left(\int_{0}^{r} \frac{s}{2} z_{\rho}^{2}(s) d s\right)^{2} d r-\int_{\frac{R}{2}}^{R} z_{\rho}(r) u(r) \sqrt{\rho} \sqrt{r}\left(\int_{0}^{r} \frac{1}{2} z_{\rho}^{2}(s) d s\right)^{2} d r \\
=\underbrace{\int_{\frac{R}{2}}^{R} z_{\rho}(r) u(r)\left(\frac{1}{r}-\frac{1}{\rho}\right)\left(\int_{0}^{r} \frac{s}{2} z_{\rho}^{2}(s) d s\right)^{2} d r}_{(I)} \\
+\underbrace{\frac{1}{\rho} \int_{\frac{R}{2}}^{R} z_{\rho}(r) u(r)\left[\left(\int_{0}^{r} \frac{s}{2} z_{\rho}^{2}(s) d s\right)^{2}-\left(\int_{0}^{r} \frac{\rho}{2} z_{\rho}^{2}(s) d s\right)^{2}\right] d r}_{(I I)} \\
+\underbrace{\sqrt{\rho} \int_{\frac{R}{2}}^{R} z_{\rho}(r) u(r)(\sqrt{\rho}-\sqrt{r})\left(\int_{0}^{r} \frac{1}{2} z_{\rho}^{2}(s) d s\right)^{2} d r}_{(I I I)} .
\end{aligned}
$$

We have

$$
\begin{aligned}
|(I)| & \leqslant \int_{\frac{R}{2}}^{R}\left|z_{\rho}(r) u(r)\right|\left|\frac{r-\rho}{r \rho}\right|\left(\int_{0}^{r} \frac{s}{2} z_{\rho}^{2}(s) d s\right)^{2} d r \\
& \leqslant C \int_{\frac{R}{2}}^{R}\left|z_{\rho}(r) u(r)\right||r-\rho| d r \\
& \leqslant C\left(\int_{\frac{R}{2}}^{R} z_{\rho}^{2}(r) \frac{(r-\rho)^{2}}{r} d r\right)^{\frac{1}{2}}\|u\| \leqslant \frac{C}{\sqrt{R}}\|u\| .
\end{aligned}
$$


Moreover

$$
\begin{aligned}
|(I I)| & \leqslant \frac{1}{\rho} \int_{\frac{R}{2}}^{R}\left|z_{\rho}(r) u(r)\right|\left|\int_{0}^{r} \frac{s-\rho}{2} z_{\rho}^{2}(s) d s\right|\left|\int_{0}^{r} \frac{s+\rho}{2} z_{\rho}^{2}(s) d s\right| d r \\
& \leqslant C \int_{\frac{R}{2}}^{R}\left|z_{\rho}(r) u(r)\right| d r \leqslant \frac{C}{\sqrt{R}}\|u\|,
\end{aligned}
$$

and

$$
\begin{aligned}
|(I I I)| & \leqslant \sqrt{\rho} \int_{\frac{R}{2}}^{R}\left|z_{\rho}(r) u(r)\right||\sqrt{\rho}-\sqrt{r}|\left(\int_{0}^{r} \frac{1}{2} z_{\rho}^{2}(s) d s\right)^{2} d r \\
& \leqslant C \sqrt{\rho} \int_{\frac{R}{2}}^{R}\left|z_{\rho}(r) u(r)\right| \frac{|\rho-r|}{\sqrt{\rho}+\sqrt{r}} d r \leqslant \frac{C}{\sqrt{R}}\|u\| .
\end{aligned}
$$

Therefore, taking also in account the exponential decay of $z_{\rho}$,

$$
\begin{aligned}
\int_{\frac{R}{2}}^{R} \frac{z_{\rho}(r) u(r)}{r}\left(\int_{0}^{r} \frac{s}{2} z_{\rho}^{2}(s) d s\right)^{2} d r= & \sqrt{\rho} \int_{\frac{R}{2}}^{R} z_{\rho}(r) u(r) \sqrt{r}\left(\int_{\frac{R}{2}}^{r} \frac{1}{2} z_{\rho}^{2}(s) d s\right)^{2} d r \\
& +O\left(R^{-1 / 2}\right)\|u\| .
\end{aligned}
$$

Analogously

$$
\begin{aligned}
& \int_{\frac{R}{2}}^{R} z_{\rho}(r) u(r) r\left(\int_{r}^{R} \frac{z_{\rho}^{2}(s)}{s}\left(\int_{0}^{s} \frac{t}{2} z_{\rho}^{2}(t) d t\right) d s\right) d r \\
& \quad-\int_{\frac{R}{2}}^{R} z_{\rho}(r) u(r) \sqrt{\rho} \sqrt{r}\left(\int_{r}^{R} z_{\rho}^{2}(s)\left(\int_{0}^{s} \frac{1}{2} z_{\rho}^{2}(t) d t\right) d s\right) d r \\
&=\int_{\frac{R}{2}}^{R} z_{\rho}(r) u(r)(r-\rho)\left(\int_{r}^{R} \frac{z_{\rho}^{2}(s)}{s}\left(\int_{0}^{s} \frac{t}{2} z_{\rho}^{2}(t) d t\right) d s\right) d r \\
& \quad+\int_{\frac{R}{2}}^{R} z_{\rho}(r) u(r)\left(\int_{r}^{R} z_{\rho}^{2}(s)\left(\frac{\rho}{s}-1\right)\left(\int_{0}^{s} \frac{t}{2} z_{\rho}^{2}(t) d t\right) d s\right) d r \\
& \quad+\int_{\frac{R}{2}}^{R} z_{\rho}(r) u(r)\left(\int_{r}^{R} z_{\rho}^{2}(s)\left(\int_{0}^{s}\left(\frac{t}{2}-\frac{\rho}{2}\right) z_{\rho}^{2}(t) d t\right) d s\right) d r \\
& \quad+\int_{\frac{R}{2}}^{R} z_{\rho}(r) u(r) \sqrt{\rho}(\sqrt{\rho}-\sqrt{r})\left(\int_{r}^{R} z_{\rho}^{2}(s)\left(\int_{0}^{s} \frac{1}{2} z_{\rho}^{2}(t) d t\right) d s\right) d r \\
&= O\left(R^{-1 / 2}\right)\|u\| .
\end{aligned}
$$

and so, again by the exponential decay of $z_{\rho}$,

$$
\begin{aligned}
\int_{\frac{R}{2}}^{R} z_{\rho}(r) u(r) r\left(\int_{r}^{R} \frac{z_{\rho}^{2}(s)}{s}\left(\int_{0}^{s} \frac{t}{2} z_{\rho}^{2}(t) d t\right) d s\right) d r \\
=\sqrt{\rho} \int_{\frac{R}{2}}^{R} z_{\rho}(r) u(r) \sqrt{r}\left(\int_{r}^{R} z_{\rho}^{2}(s)\left(\int_{\frac{R}{2}}^{s} \frac{1}{2} z_{\rho}^{2}(t) d t\right) d s\right) d r \\
\quad+O\left(R^{-1 / 2}\right)\|u\| .
\end{aligned}
$$


Then, by (24), (25), (26), (27) and (28), we get (23).

Let us observe that

$$
\begin{aligned}
\sqrt{\rho} \int_{\frac{R}{2}}^{R} z_{\rho}^{\prime} u^{\prime} \sqrt{r} d r & =-\sqrt{\rho} \int_{\frac{R}{2}}^{R} z_{\rho}^{\prime \prime} u \sqrt{r} d r-\sqrt{\rho} \int_{\frac{R}{2}}^{R} \frac{z_{\rho}^{\prime}}{2 \sqrt{r}} u d r+o\left(R^{-1 / 2}\right)\|u\| \\
& =-\sqrt{\rho} \int_{\frac{R}{2}}^{R} z_{\rho}^{\prime \prime} u \sqrt{r} d r+O\left(R^{-1 / 2}\right)\|u\| .
\end{aligned}
$$

Therefore, by (19) and (20), we have

$$
\begin{aligned}
& \frac{I_{R}^{\prime}\left(z_{\rho}\right)[u]}{2 \pi}=\sqrt{\rho} \int_{\frac{R}{2}}^{R}\left[-z_{\rho}^{\prime \prime}+\omega z_{\rho}+\frac{z_{\rho}}{4}\left[\left(\int_{\frac{R}{2}}^{r} z_{\rho}^{2}(s) d s\right)^{2}+2\left(\int_{r}^{R} z_{\rho}^{2}(s)\left(\int_{\frac{R}{2}}^{s} z_{\rho}^{2}(t) d t\right) d s\right)\right]-z_{\rho}^{p}\right] u \sqrt{r} d r \\
& +O\left(R^{-1 / 2}\right)\|u\| \\
& =\sqrt{\rho} \int_{\frac{R}{2}}^{R}\left[-z_{\rho}^{\prime \prime}+\omega z_{\rho}+\frac{z_{\rho}}{4}\left(\int_{\frac{R}{2}}^{R} z_{\rho}^{2}(s) d s\right)^{2}-z_{\rho}^{p}\right] u \sqrt{r} d r \\
& +O\left(R^{-1 / 2}\right)\|u\| \\
& =\underbrace{\frac{\sqrt{\rho}}{4}\left[\left(\int_{\frac{R}{2}}^{R} U_{\rho}^{2}(s) d s\right)^{2}-\left(\int_{-\infty}^{+\infty} U^{2}(s) d s\right)^{2}\right] \int_{\frac{R}{2}}^{R} U_{\rho} u \sqrt{r} d r}_{(A)} \\
& -\underbrace{\frac{\sqrt{\rho}}{4}\left[\left(\int_{\frac{R}{2}}^{R} U_{\rho}^{2}(s) d s\right)^{2}-\left(\int_{-\infty}^{+\infty} U^{2}(s) d s\right)^{2}\right] \int_{\frac{R}{2}}^{R} V_{\rho} u \sqrt{r} d r}_{(B)} \\
& -\underbrace{\sqrt{\rho}\left(\int_{\frac{R}{2}}^{R} U_{\rho}^{2}(s) d s\right)\left(\int_{\frac{R}{2}}^{R} U_{\rho}(s) V_{\rho}(s) d s\right) \int_{\frac{R}{2}}^{R} U_{\rho} u \sqrt{r} d r}_{(C)} \\
& +\underbrace{\frac{\sqrt{\rho}}{2}\left(\int_{\frac{R}{2}}^{R} U_{\rho}^{2}(s) d s\right)\left(\int_{\frac{R}{2}}^{R} V_{\rho}^{2}(s) d s\right) \int_{\frac{R}{2}}^{R} U_{\rho} u \sqrt{r} d r}_{(D)} \\
& -\underbrace{\sqrt{\rho} \int_{\frac{R}{2}}^{R}\left[\left(U_{\rho}-V_{\rho}\right)^{p}-U_{\rho}^{p}\right] u \sqrt{r} d r}_{(E)} \\
& +o\left(e^{-2 \sqrt{k_{2}}(R-\rho)}\right)\|u\| .
\end{aligned}
$$

Let us evaluate every term in the previous formula. Since

$$
\left(\int_{\frac{R}{2}}^{R} U_{\rho}^{2}(s) d s\right)^{2}-\left(\int_{-\infty}^{+\infty} U^{2}(s) d s\right)^{2}=O\left(e^{-2 \sqrt{k_{2}}(R-\rho)}\right)
$$

we infer that

$$
\begin{aligned}
& (A)=O\left(\sqrt{\rho} e^{-2 \sqrt{k_{2}}(R-\rho)}\right)\|u\|, \\
& (B)=o\left(\sqrt{\rho} e^{-2 \sqrt{k_{2}}(R-\rho)}\right)\|u\| .
\end{aligned}
$$


Moreover, by [1, Lemma 3.7],

$$
(C)=O\left(\sqrt{\rho}|R-\rho| e^{-2 \sqrt{k_{2}}(R-\rho)}\right)\|u\| .
$$

The estimate of $(D)$ is direct:

$$
(D)=O\left(\sqrt{\rho} e^{-2 \sqrt{k_{2}}(R-\rho)}\right)\|u\| .
$$

Finally, by the Mean Value Theorem we can estimate:

$$
\left|\left(U_{\rho}-V_{\rho}\right)^{p}-U_{\rho}^{p}\right| \leqslant C U_{\rho}^{p-1} V_{\rho} .
$$

Again by [1, Lemma 3.7], we have

$$
\begin{aligned}
(E) & \leqslant \sqrt{\rho}\left(\int_{\frac{R}{2}}^{R}\left[\left(U_{\rho}-V_{\rho}\right)^{p}-U_{\rho}^{p}\right]^{2} d r\right)^{\frac{1}{2}}\|u\| \leqslant \sqrt{\rho}\left(\int_{\frac{R}{2}}^{R} U_{\rho}^{2(p-1)} V_{\rho}^{2} d r\right)^{\frac{1}{2}}\|u\| \\
& = \begin{cases}O\left(\sqrt{\rho} e^{-2 \sqrt{k_{2}}(R-\rho)}\right)\|u\|, & \text { if } p>2, \\
O\left(\sqrt{\rho}|R-\rho|^{\frac{1}{2}} e^{-2 \sqrt{k_{2}}(R-\rho)}\right)\|u\|, & \text { if } p=2, \\
O\left(\sqrt{\rho} e^{-p \sqrt{k_{2}}(R-\rho)}\right)\|u\|, & \text { if } p<2 .\end{cases}
\end{aligned}
$$

Hence we get the conclusion.

Our next result concerns the non-degeneracy of $I_{R}^{\prime \prime}\left(z_{\rho}\right)$, and makes use of Corollary 2.5 in an essential way.

Lemma 3.2. There exist $C>c>0$ such that for large $R$ and for any $\rho \in \mathcal{I}_{R}$,

$$
c\|u\|^{2} \leqslant I_{R}^{\prime \prime}\left(z_{\rho}\right)[u, u] \leqslant C\|u\|^{2}, \quad \text { for all } u \perp T_{z_{\rho}} Z .
$$

Proof. Let $u \in H_{R}$ such that $u \perp T_{z_{\rho}} Z$. Take $a \in[R-2 \sqrt{R}, R-\sqrt{R}]$ such that

$$
\int_{a}^{a+1}\left(\left|u^{\prime}\right|^{2}+\omega u^{2}\right) r d r \leqslant \frac{2}{\sqrt{R}}\|u\|^{2} .
$$

Take $\psi$ a cut-off function such that

$$
\psi(r)= \begin{cases}0 & \text { if } 0 \leqslant r \leqslant a \\ (r-a) & \text { if } a \leqslant r \leqslant a+1 \\ 1 & \text { if } r \geqslant a+1\end{cases}
$$

Since $u=\psi u+(1-\psi) u$, we have

$$
\|u\|^{2}=\|\psi u\|^{2}+\|(1-\psi) u\|^{2}+2\langle\psi u,(1-\psi) u\rangle .
$$

Moreover

$I_{R}^{\prime \prime}\left(z_{\rho}\right)[u, u]=I_{R}^{\prime \prime}\left(z_{\rho}\right)[\psi u, \psi u]+I_{R}^{\prime \prime}\left(z_{\rho}\right)[(1-\psi) u,(1-\psi) u]+2 I_{R}^{\prime \prime}\left(z_{\rho}\right)[\psi u,(1-\psi) u]$.

For that sake of brevity, we set $v=\psi u$ and $w=(1-\psi) u$. By (35), we infer that

$$
\begin{aligned}
I_{R}^{\prime \prime}\left(z_{\rho}\right)[v, w] & =\langle v, w\rangle+o(1)\|u\|=o(1)\|u\|, \\
I_{R}^{\prime \prime}\left(z_{\rho}\right)[w, w] & =\|w\|^{2}+o(1)\|u\| .
\end{aligned}
$$

Before evaluating $I_{R}^{\prime \prime}\left(z_{\rho}\right)[v, v]$, let us observe that since $u \perp T_{z_{\rho}} Z$, then

$$
\left|\left\langle v, \dot{z}_{\rho}\right\rangle\right|=\left|-\left\langle w, \dot{z}_{\rho}\right\rangle\right|=o\left(e^{-R^{1 / 3}}\right)\|u\| .
$$

We now extend $v$ to 0 in all $\mathbb{R}$, and estimate $\left\langle v, U_{\rho}^{\prime}\right\rangle_{H^{1}(\mathbb{R})}$. On that purpose, it is easy to see that:

$$
\rho\left\langle v, U_{\rho}^{\prime}\right\rangle_{H^{1}(\mathbb{R})}-\left\langle v, U_{\rho}^{\prime}\right\rangle=O\left(R^{-1 / 2}\right)\|v\| .
$$


Moreover, by using (22),

$$
\left\langle v, \dot{z}_{\rho}+U_{\rho}^{\prime}\right\rangle=U^{\prime}(R-\rho)\left\langle v, e^{\sqrt{k_{2}}(\cdot-R)}\right\rangle=\left|U^{\prime}(R-\rho)\right| O(\sqrt{R})\|v\| .
$$

Putting together (31), (32) and (33), we conclude that

$$
\left\langle v, U_{\rho}^{\prime}\right\rangle_{H^{1}(\mathbb{R})}=o\left(R^{-1 / 2}\right)\|v\|
$$

We now study $I_{R}^{\prime \prime}\left(z_{\rho}\right)[v, v]$; here we will derive $I_{R}^{\prime \prime}$ from expression (8), obtaining the expression:

$$
\begin{aligned}
\frac{I_{R}^{\prime \prime}\left(z_{\rho}\right)[u, v]}{2 \pi}= & \int_{0}^{R}\left(u^{\prime} v^{\prime}+\omega u v\right) r d r+\int_{0}^{R} \frac{u(r) v(r)}{r}\left(\int_{0}^{r} \frac{s}{2} z_{\rho}^{2}(s) d s\right)^{2} d r \\
& +\int_{0}^{R} \frac{z_{\rho}(r) u(r)}{r}\left(\int_{0}^{r} s z_{\rho}^{2}(s) d s\right)\left(\int_{0}^{r} s z_{\rho}(s) v(s) d s\right) d r \\
& +\int_{0}^{R} u(r) v(r) r\left(\int_{r}^{R} \frac{z_{\rho}^{2}(s)}{s}\left(\int_{0}^{s} \frac{t}{2} z_{\rho}^{2}(t) d t\right) d s\right) d r \\
& +\int_{0}^{R} z_{\rho}(r) u(r) r\left(\int_{r}^{R} \frac{z_{\rho}(s) v(s)}{s}\left(\int_{0}^{s} t z_{\rho}^{2}(t) d t\right) d s\right) d r \\
& +\int_{0}^{R} z_{\rho}(r) u(r) r\left(\int_{r}^{R} \frac{z_{\rho}^{2}(s)}{s}\left(\int_{0}^{s} t z_{\rho}(t) v(t) d t\right) d s\right) d r \\
& -p \int_{0}^{R} z_{\rho}^{p-1} u v r d r .
\end{aligned}
$$

Arguing as in the proof of Lemma 3.1, we can substitute $r, s, t$ with $\rho$ committing negligible errors: more precisely, we have

$$
\begin{aligned}
\frac{I_{R}^{\prime \prime}\left(z_{\rho}\right)[v, v]}{2 \pi}= & \rho \int_{0}^{R}\left(\left(v^{\prime}\right)^{2}+\omega v^{2}\right) d r+\frac{\rho}{4} \int_{0}^{R} v^{2}(r)\left(\int_{0}^{r} z_{\rho}^{2}(s) d s\right)^{2} d r \\
& +\rho \int_{0}^{R} z_{\rho}(r) v(r)\left(\int_{0}^{r} z_{\rho}^{2}(s) d s\right)\left(\int_{0}^{r} z_{\rho}(s) v(s) d s\right) d r \\
& +\frac{\rho}{2} \int_{0}^{R} v^{2}(r)\left(\int_{r}^{R} z_{\rho}^{2}(s)\left(\int_{0}^{s} z_{\rho}^{2}(t) d t\right) d s\right) d r \\
& +\rho \int_{0}^{R} z_{\rho}(r) v(r)\left(\int_{r}^{R} z_{\rho}(s) v(s)\left(\int_{0}^{s} z_{\rho}^{2}(t) d t\right) d s\right) d r \\
& +\rho \int_{0}^{R} z_{\rho}(r) v(r)\left(\int_{r}^{R} z_{\rho}^{2}(s)\left(\int_{0}^{s} z_{\rho}(t) v(t) d t\right) d s\right) d r \\
& -\rho p \int_{0}^{R} z_{\rho}^{p-1} v^{2} d r+o(1)\|u\|^{2} .
\end{aligned}
$$

Let us observe that,

$$
\begin{aligned}
& \rho \int_{0}^{R}\left(\left(v^{\prime}\right)^{2}+\omega v^{2}\right) d r-\rho p \int_{0}^{R} z_{\rho}^{p-1} v^{2} d r \\
&=\rho \int_{-\infty}^{+\infty}\left(\left(v^{\prime}\right)^{2}+\omega v^{2}\right) d r-\rho p \int_{-\infty}^{+\infty} U_{\rho}^{p-1} v^{2} d r+o(1)\|u\|^{2} .
\end{aligned}
$$


Setting $F(r)=\int_{0}^{r} z_{\rho}^{2}(s) d s$, since $F^{\prime}(r)=z_{\rho}^{2}(r)$, we get

$$
\begin{aligned}
\frac{\rho}{4} \int_{0}^{R} v^{2}(r) & \left(\int_{0}^{r} z_{\rho}^{2}(s) d s\right)^{2} d r+\frac{\rho}{2} \int_{0}^{R} v^{2}(r)\left(\int_{r}^{R} z_{\rho}^{2}(s)\left(\int_{0}^{s} z_{\rho}^{2}(t) d t\right) d s\right) d r \\
= & \frac{\rho}{4}\left(\int_{0}^{R} z_{\rho}^{2}(r) d r\right)^{2}\left(\int_{0}^{R} v^{2}(r) d r\right) \\
= & \frac{\rho}{4}\left(\int_{-\infty}^{+\infty} U_{\rho}^{2}(r) d r\right)^{2}\left(\int_{-\infty}^{+\infty} v^{2}(r) d r\right)+o(1)\|u\|^{2} .
\end{aligned}
$$

Setting, moreover, $G(r)=\int_{0}^{r} z_{\rho}(s) v(s) d s$, since $G^{\prime}(r)=z_{\rho}(r) v(r)$, we have

$$
\begin{aligned}
\rho \int_{0}^{R} z_{\rho}(r) & v(r)\left(\int_{0}^{r} z_{\rho}^{2}(s) d s\right)\left(\int_{0}^{r} z_{\rho}(s) v(s) d s\right) d r \\
& +\rho \int_{0}^{R} z_{\rho}(r) v(r)\left(\int_{r}^{R} z_{\rho}(s) v(s)\left(\int_{0}^{s} z_{\rho}^{2}(t) d t\right) d s\right) d r \\
& +\rho \int_{0}^{R} z_{\rho}(r) v(r)\left(\int_{r}^{R} z_{\rho}^{2}(s)\left(\int_{0}^{s} z_{\rho}(t) v(t) d t\right) d s\right) d r \\
= & \left(\int_{0}^{R} z_{\rho}^{2}(r) d r\right)\left(\int_{0}^{R} z_{\rho}(r) v(r) d r\right)^{2} \\
= & \rho\left(\int_{-\infty}^{+\infty} U_{\rho}^{2}(r) d r\right)\left(\int_{-\infty}^{+\infty} U_{\rho}(r) v(r) d r\right)^{2}+o(1)\|u\|^{2}
\end{aligned}
$$

Therefore, by (34):

$$
I_{R}^{\prime \prime}\left(z_{\rho}\right)[v, v]=\rho L[v, v]+o(1)\|u\|^{2} .
$$

By Corollary 2.5 we have that $c\|v\|_{H^{1}(\mathbb{R})}^{2} \leqslant L[v, v] \leqslant C\|v\|_{H^{1}(\mathbb{R})}^{2}$. Observe moreover that $\rho\|v\|_{H^{1}(\mathbb{R})}^{2}=\|v\|^{2}+o(1)\|v\|^{2}$. These estimates, together with (29) and (30), yield the conclusion.

Our next estimate implies that the previous non-degeneracy result applies also for $I_{R}^{\prime \prime}\left(z_{\rho}+w\right)$ if $w$ is sufficiently small.

Lemma 3.3. There exists $C>0$ such that

$$
\left|I_{R}^{\prime \prime}\left(z_{\rho}\right)[u, u]-I_{R}^{\prime \prime}\left(z_{\rho}+w\right)[u, u]\right| \leqslant C\left(\|w\|+\|w\|^{p-1}\right)\|u\|^{2},
$$

for all $u, w \in H_{R}, R$ sufficiently large and $\rho \in \mathcal{I}_{R}$.

Proof. For this estimate, we use the form of $I_{R}^{\prime \prime}\left(z_{\rho}\right)$ derived from (7), that is:

$$
\begin{aligned}
\frac{I_{R}^{\prime \prime}\left(z_{\rho}\right)[u, u]}{2 \pi}= & \int_{0}^{R}\left(u^{\prime 2}+\omega u^{2}\right) r d r-p \int_{0}^{R} z_{\rho}^{p-1} u^{2} r d r \\
& +\frac{1}{2} \int_{0}^{R} \frac{z_{\rho}^{2}(r)}{r}\left(\int_{0}^{r} s z_{\rho}^{2}(s) d s\right)\left(\int_{0}^{r} u^{2}(s) s d s\right) d r \\
& +2 \int_{0}^{R} \frac{z_{\rho}(r) u(r)}{r}\left(\int_{0}^{r} s z_{\rho}(s) u(s) d s\right)\left(\int_{0}^{r} s z_{\rho}^{2}(s) d s\right) d r \\
& +\frac{1}{4} \int_{0}^{R} \frac{u^{2}(r)}{r}\left(\int_{0}^{r} s z_{\rho}^{2}(s) d s\right)^{2} d r+\int_{0}^{R} \frac{z_{\rho}^{2}(r)}{r}\left(\int_{0}^{r} s z_{\rho}(s) u(s) d s\right)^{2} d r .
\end{aligned}
$$


For the estimate of the local term, we use the inequalities:

$$
|a+b|^{q}-|a|^{q} \leqslant\left\{\begin{array}{ll}
C\left(|a|^{q-1}+|b|^{q-1}\right)|b| & \text { if } q>1, \\
|b|^{q} & \text { if } q \in(0,1]
\end{array}, a, b \geqslant 0 .\right.
$$

Then, if $p>2$, we use the $L^{\infty}$ bound of $z_{\rho}$ to get:

$$
\begin{aligned}
& \int_{0}^{R}\left(\left|z_{\rho}+\right| w||^{p-1}-\left|z_{\rho}\right|^{p-1}\right) u^{2} r d r \leqslant C \int_{0}^{R}\left(|w|+|w|^{p-1}\right) u^{2} r d r \\
& \leqslant C\left(\int_{0}^{R}\left(|w|^{2}+|w|^{2(p-1)}\right) r d r\right)^{1 / 2}\left(\int_{0}^{R} u^{4} r d r\right)^{1 / 2} .
\end{aligned}
$$

If $p \in(1,2]$,

$$
\begin{array}{rl}
\int_{0}^{R}\left(\left|z_{\rho}+\right| w||^{p-1}-\left|z_{\rho}\right|^{p-1}\right) u^{2} & d r \leqslant \int_{0}^{R}|w|^{p-1} u^{2} r d r \\
& \leqslant\left(\int_{0}^{R}|w|^{2} r d r\right)^{\frac{p-1}{2}}\left(\int_{0}^{R}|u(r)|^{\frac{4}{3-p}} r d r\right)^{\frac{3-p}{2}} .
\end{array}
$$

Let us now consider the nonlocal terms. Observe that each of those terms is of order 4 in $z_{\rho}$. The estimates of each term is relatively easy, but the whole computation is lengthy. Let us explain in detail some of them. For instance, let us define:

$$
Q\left(z_{1}, z_{2}, z_{3}, z_{4}\right)=\int_{0}^{R} \frac{z_{1}(r) z_{2}(r)}{r}\left(\int_{0}^{r} s z_{3}(s) z_{4}(s) d s\right)\left(\int_{0}^{r} s u^{2}(s) d s\right) d r .
$$

Clearly $Q$ is linear in each variable. Therefore, we need to estimate:

$$
Q\left(z_{1}, z_{2}, z_{3}, z_{4}\right) \quad \text { where } z_{i}=w \forall i \in A, \quad z_{i}=z_{\rho} \forall i \notin A,
$$

for any non-empty $A \subset\{1,2,3,4\}$. For instance, we estimate:

$$
\begin{aligned}
Q\left(z_{\rho}, z_{\rho}, z_{\rho}, w\right) & =\int_{0}^{R} \frac{z_{\rho}^{2}}{r}\left(\int_{0}^{r} s z_{\rho}(s) w(s) d s\right)\left(\int_{0}^{r} u^{2}(s) s d s\right) d r \\
& \leqslant \int_{0}^{R} \frac{z_{\rho}^{2}}{\sqrt{r}} d r \int_{0}^{R} z_{\rho}(s) w(s) \sqrt{s} d s \int_{0}^{R} u^{2}(s) s d s \\
& \leqslant \int_{0}^{R} \frac{z_{\rho}^{2}}{\sqrt{r}} d r \cdot\left(\int_{0}^{R} z_{\rho}^{2} d r\right)^{\frac{1}{2}}\|w\|_{L^{2}}\|u\|^{2} \leqslant \frac{C}{\sqrt{R}}\|w\|_{L^{2}}\|u\|_{L^{2}}^{2} .
\end{aligned}
$$

The cases where $w$ appears more than once are easier.

Slightly more difficult is the estimate of $K\left(z_{\rho}, z_{\rho}, z_{\rho}, w\right)$, with

$$
K\left(z_{1}, z_{2}, z_{3}, z_{4}\right)=\int_{0}^{R} \frac{z_{1}(r) u(r)}{r}\left(\int_{0}^{r} s z_{2}(s) u(s) d s\right)\left(\int_{0}^{r} s z_{3}(s) z_{4}(s) d s\right) d r
$$

Indeed, in this case $z_{\rho}$ appears in the three integrals. Then, we need to share the variable, as follows:

$$
\begin{aligned}
K\left(z_{\rho}, z_{\rho}, z_{\rho}, w\right) & =\int_{0}^{R} \frac{z_{\rho}(r) u(r)}{r}\left(\int_{0}^{r} s z_{\rho}(s) u(s) d s\right)\left(\int_{0}^{r} s z_{\rho}(s) w(s) d s\right) d r \\
& \leqslant \int_{0}^{R} \frac{z_{\rho}(r)}{\sqrt{r}} u(r) \sqrt{r} d r \int_{0}^{R} z_{\rho}(s) u(s) \sqrt{s} d s \int_{0}^{R} z_{\rho}(s) w(s) \sqrt{s} d s d r \\
& \leqslant \frac{C}{\sqrt{R}}\|u\|_{L^{2}}^{2}\|w\|_{L^{2}} .
\end{aligned}
$$


The other terms can be estimated in an analogous way.

We are now in conditions of stating the main result of this section.

Proposition 3.4. For sufficiently large $R$ and any $\rho \in \mathcal{I}_{R}$, there exists a unique $w_{\rho} \in H_{R}$ such that:

(1) $z_{\rho}+w_{\rho}$ solves the auxiliary equation $P I_{R}^{\prime}\left(z_{\rho}+w_{\rho}\right)=0$.

(2) The map $\rho \mapsto w_{\rho}$ is $C^{1}$.

(3)

$$
\left\|w_{\rho}\right\|= \begin{cases}O\left(R^{\frac{1-2 \alpha}{2}} \log R\right), & \text { if } p \geqslant 2, \\ O\left(R^{\frac{1-p \alpha}{2}}\right), & \text { if } p<2 .\end{cases}
$$

Proof. The proof is quite standard in Lyapunov-Schmidt reduction. By Lemma 3.1, and taking into account (21), we obtain that $I_{R}^{\prime}\left(z_{\rho}\right)=o(1)$. This, together with the estimates of Lemmas 3.2, 3.3, makes it possible to carry out the contraction argument. Moreover, the $C^{1}$ regularity of the map $\rho \mapsto w_{\rho}$ can be deduced from the Implicit Function Theorem. See [2], Sections 2.3, 2.4, for details.

Moreover, by taking into account that $\rho \in \mathcal{I}_{R}$, we conclude that:

$$
\left\|I_{R}^{\prime}\left(z_{\rho}\right)\right\|= \begin{cases}O\left(R^{\frac{1-2 \alpha}{2}} \log R\right), & \text { if } p \geqslant 2, \\ O\left(R^{\frac{1-p \alpha}{2}}\right), & \text { if } p<2 .\end{cases}
$$

Therefore, the same estimate for $w_{\rho}$ holds.

Remark 3.5. It is important to observe that most of the results of this section hold with the same proof if we consider $\omega \in\left(0, \omega_{1}\right)$, and also if we take $U=w_{k_{1}}$ instead of $w_{k_{2}}$ (Lemma 3.2 should be restated for $U=w_{k_{1}}$, since this is not a minimizer for $J$ ). In next section, though, we will obtain a solution of the bifurcation equation only if $J(U)<0$. This is the reason why we need $\omega \in\left(0, \omega_{0}\right), U=w_{k_{2}}$, according to Proposition 2.2. But those restrictions are natural, as commented in the Introduction.

\section{THE REDUCED FUNCTIONAL}

Let us define:

$$
\tilde{Z}=\left\{z_{\rho}+w_{\rho}: \rho \in \mathcal{I}_{R}, w_{\rho} \text { as in Proposition 3.4 }\right\} .
$$

It is well-known (see [2]) that $\tilde{Z}$ is a natural constraint for $I_{R}$ or, in other words, that critical points of $\left.I_{R}\right|_{\tilde{Z}}$ correspond to solutions of the bifurcation equation, and hence they are true critical points of $I_{R}$.

This section is devoted to prove the existence of a minimum for $\left.I_{R}\right|_{\tilde{Z}}$, which receives the name of reduced functional.

Lemma 4.1. For any $\rho \in \mathcal{I}_{R}$, we have

$$
I_{R}\left(z_{\rho}\right)=2 \pi J(U) \rho+2 \pi \sqrt{k_{2}} \rho e^{-2 \sqrt{k_{2}}(R-\rho)}+o\left(\rho e^{-2 \sqrt{k_{2}}(R-\rho)}\right) .
$$

Proof. We have

$$
\begin{aligned}
\frac{I_{R}\left(z_{\rho}\right)}{2 \pi}= & \frac{1}{2} \int_{0}^{R}\left(\left|z_{\rho}^{\prime}\right|^{2}+\omega z_{\rho}^{2}\right) r d r+\frac{1}{8} \int_{0}^{R} \frac{z_{\rho}^{2}(r)}{r}\left(\int_{0}^{r} s z_{\rho}^{2}(s) d s\right)^{2} d r \\
& -\frac{1}{p+1} \int_{0}^{R} z_{\rho}^{p+1} r d r .
\end{aligned}
$$


BOUNDARY CONCENTRATION OF A GAUGED NONLINEAR SCHRÖDINGER EQUATION

19

Let us evaluate all these integrals in $[0, R / 2]$. We have

$$
\left.\left|\int_{0}^{\frac{R}{2}}\right| z_{\rho}^{\prime}\right|^{2} r d r \mid \leqslant C R e^{2 \sqrt{k_{2}}\left(\frac{R}{2}-\rho\right)} \leqslant C R e^{-\sqrt{k_{2}} \frac{R}{2}}
$$

Analogously,

$$
\begin{gathered}
\int_{0}^{\frac{R}{2}} \omega z_{\rho}^{2} r d r \leqslant C R e^{-\sqrt{k_{2}} \frac{R}{2}} \\
\int_{0}^{\frac{R}{2}} \frac{z_{\rho}^{2}(r)}{r}\left(\int_{0}^{r} s z_{\rho}^{2}(s) d s\right)^{2} d r \leqslant C R e^{-\sqrt{k_{2}} \frac{3 R}{2}} \\
\int_{0}^{\frac{R}{2}} z_{\rho}^{p+1} r d r \leqslant C R e^{-(p+1) \sqrt{k_{2}} \frac{R}{4}}
\end{gathered}
$$

Therefore

$$
\begin{aligned}
\frac{I_{R}\left(z_{\rho}\right)}{2 \pi}= & \frac{1}{2} \int_{\frac{R}{2}}^{R}\left(\left|z_{\rho}^{\prime}\right|^{2}+\omega z_{\rho}^{2}\right) r d r+\frac{1}{8} \int_{\frac{R}{2}}^{R} \frac{z_{\rho}^{2}(r)}{r}\left(\int_{0}^{r} s z_{\rho}^{2}(s) d s\right)^{2} d r \\
& -\frac{1}{p+1} \int_{\frac{R}{2}}^{R} z_{\rho}^{p+1} r d r+o\left(e^{-\sqrt{k_{2}} \frac{R}{5}}\right) .
\end{aligned}
$$

Let us check that

(36)

$$
\frac{I_{R}\left(z_{\rho}\right)}{2 \pi}=\rho\left[\frac{1}{2} \int_{\frac{R}{2}}^{R}\left(\left|z_{\rho}^{\prime}\right|^{2}+\omega z_{\rho}^{2}\right) d r+\frac{1}{24}\left(\int_{\frac{R}{2}}^{R} z_{\rho}^{2} d r\right)^{3}-\frac{1}{p+1} \int_{\frac{R}{2}}^{R} z_{\rho}^{p+1} d r\right]+O(1) .
$$

Indeed

$$
\left.\left|\int_{\frac{R}{2}}^{R}\right| z_{\rho}^{\prime}\right|^{2} r d r-\left.\rho \int_{\frac{R}{2}}^{R}\left|z_{\rho}^{\prime}\right|^{2} d r\left|\leqslant \int_{\frac{R}{2}}^{R}\right| z_{\rho}^{\prime}(r)\right|^{2}|r-\rho| d r \leqslant C,
$$

and hence

$$
\int_{\frac{R}{2}}^{R}\left|z_{\rho}^{\prime}\right|^{2} r d r=\rho \int_{\frac{R}{2}}^{R}\left|z_{\rho}^{\prime}\right|^{2} d r+O(1)
$$

Analogously

$$
\begin{gathered}
\int_{\frac{R}{2}}^{R} z_{\rho}^{2} r d r=\rho \int_{\frac{R}{2}}^{R} z_{\rho}^{2} d r+O(1), \\
\int_{\frac{R}{2}}^{R} z_{\rho}^{p+1} r d r=\rho \int_{\frac{R}{2}}^{R} z_{\rho}^{p+1} d r+O(1) .
\end{gathered}
$$

Moreover

$$
\begin{gathered}
\int_{\frac{R}{2}}^{R} \frac{z_{\rho}^{2}(r)}{r}\left(\int_{0}^{r} s z_{\rho}^{2}(s) d s\right)^{2} d r-\rho \int_{\frac{R}{2}}^{R} z_{\rho}^{2}(r)\left(\int_{0}^{r} z_{\rho}^{2}(s) d s\right)^{2} d r \\
=\underbrace{\int_{\frac{R}{2}}^{R} z_{\rho}^{2}(r)\left(\frac{1}{r}-\frac{1}{\rho}\right)\left(\int_{0}^{r} s z_{\rho}^{2}(s) d s\right)^{2} d r}_{(I)} \\
+\underbrace{\frac{1}{\rho} \int_{\frac{R}{2}}^{R} z_{\rho}^{2}(r)\left[\left(\int_{0}^{r} s z_{\rho}^{2}(s) d s\right)^{2}-\left(\int_{0}^{r} \rho z_{\rho}^{2}(s) d s\right)^{2}\right] d r}_{(I I)} .
\end{gathered}
$$


Since

$$
\begin{aligned}
& |(I)| \leqslant C \int_{\frac{R}{2}}^{R} z_{\rho}^{2}(r)|r-\rho|\left(\int_{\frac{R}{2}}^{R} z_{\rho}^{2}(s) d s\right)^{2} d r \leqslant C . \\
& |(I I)| \leqslant \frac{C}{\rho} \int_{\frac{R}{2}}^{R} z_{\rho}(r)^{2}\left|\int_{0}^{r} \frac{s-\rho}{2} z_{\rho}^{2}(s) d s\right|\left|\int_{0}^{r} \frac{s+\rho}{2} z_{\rho}^{2}(s) d s\right| d r \leqslant C,
\end{aligned}
$$

we have

$$
\begin{aligned}
\int_{\frac{R}{2}}^{R} \frac{z_{\rho}^{2}(r)}{r}\left(\int_{0}^{r} s z_{\rho}^{2}(s) d s\right)^{2} d r & =\rho \int_{\frac{R}{2}}^{R} z_{\rho}^{2}(r)\left(\int_{\frac{R}{2}}^{r} z_{\rho}^{2}(s) d s\right)^{2} d r+O(1) \\
& =\frac{\rho}{3}\left(\int_{\frac{R}{2}}^{R} z_{\rho}^{2}(r) d r\right)^{3}+O(1) .
\end{aligned}
$$

Hence (36) follows by (37), (38), (39) and (40).

By (36), we infer that

$$
\begin{aligned}
& \frac{I_{R}\left(z_{\rho}\right)}{2 \pi}=\rho[\underbrace{\frac{1}{2} \int_{\frac{R}{2}}^{R}\left(\left|U_{\rho}^{\prime}\right|^{2}+\omega U_{\rho}^{2}\right) d r+\frac{1}{24}\left(\int_{\frac{R}{2}}^{R} U_{\rho}^{2} d r\right)^{3}-\frac{1}{p+1} \int_{\frac{R}{2}}^{R} U_{\rho}^{p+1} d r}_{(A)} \\
& \underbrace{-\int_{\frac{R}{2}}^{R}\left(U_{\rho}^{\prime} V_{\rho}^{\prime}+\omega U_{\rho} V_{\rho}\right) d r-\frac{1}{4}\left(\int_{\frac{R}{2}}^{R} U_{\rho}^{2} d r\right)^{2} \int_{\frac{R}{2}}^{R} U_{\rho} V_{\rho} d r+\int_{\frac{R}{2}}^{R} U_{\rho}^{p} V_{\rho} d r}_{(B)} \\
& \underbrace{+\frac{1}{2} \int_{\frac{R}{2}}^{R}\left(\left|V_{\rho}^{\prime}\right|^{2}+\omega V_{\rho}^{2}\right) d r+\frac{1}{8}\left(\int_{\frac{R}{2}}^{R} U_{\rho}^{2} d r\right)^{2} \int_{\frac{R}{2}}^{R} V_{\rho}^{2} d r}_{(C)} \\
& \underbrace{-\frac{1}{p+1} \int_{\frac{R}{2}}^{R}\left(\left(U_{\rho}-V_{\rho}\right)^{p+1}-U_{\rho}^{p+1}+(p+1) U_{\rho}^{p} V_{\rho}\right) d r}_{(D)}] \\
& +O\left(\rho|R-\rho|^{2} e^{-4 \sqrt{k_{2}}(R-\rho)}\right) \text {. }
\end{aligned}
$$

We have to evaluate each term of the previous formula. Firstly, let us observe that

$$
\int_{\frac{R}{2}}^{R} U_{\rho}^{2} d r=\int_{-\infty}^{+\infty} U^{2} d r-\frac{e^{-2 \sqrt{k_{2}}(R-\rho)}}{2 \sqrt{k_{2}}}+o\left(e^{-2 \sqrt{k_{2}}(R-\rho)}\right) .
$$


Since $U_{\rho}$ is a solution of (19), we have

$$
\begin{aligned}
(A)= & \frac{1}{2} \int_{\frac{R}{2}}^{R}\left(-U_{\rho}^{\prime \prime}+\omega U_{\rho}\right) U_{\rho} d r+\frac{1}{24}\left(\int_{\frac{R}{2}}^{R} U_{\rho}^{2} d r\right)^{3}-\frac{1}{p+1} \int_{\frac{R}{2}}^{R} U_{\rho}^{p+1} d r \\
& +\frac{1}{2} U_{\rho}(R) U_{\rho}^{\prime}(R)+o\left(e^{-\sqrt{k_{2}} \frac{R}{4}}\right) \\
= & -\frac{1}{8}\left(\int_{-\infty}^{+\infty} U^{2} d r\right)^{2} \int_{\frac{R}{2}}^{R} U_{\rho}^{2} d r+\frac{1}{24}\left(\int_{\frac{R}{2}}^{R} U_{\rho}^{2} d r\right)^{3}+\left(\frac{1}{2}-\frac{1}{p+1}\right) \int_{\frac{R}{2}}^{R} U_{\rho}^{p+1} d r \\
& -\frac{1}{2} \sqrt{k_{2}} e^{-2 \sqrt{k_{2}}(R-\rho)}+o\left(e^{-\sqrt{k_{2}} \frac{R}{4}}\right) \\
= & -\frac{1}{12}\left(\int_{-\infty}^{+\infty} U^{2} d r\right)^{3}+\left(\frac{1}{2}-\frac{1}{p+1}\right) \int_{-\infty}^{+\infty} U^{p+1} d r-\frac{1}{2} \sqrt{k_{2}} e^{-2 \sqrt{k_{2}}(R-\rho)} \\
& +o\left(e^{-2 \sqrt{k_{2}}(R-\rho)}\right) \\
= & J(U)-\frac{1}{2} \sqrt{k_{2}} e^{-2 \sqrt{k_{2}}(R-\rho)}+o\left(e^{-2 \sqrt{k_{2}}(R-\rho)}\right) .
\end{aligned}
$$

Again, being $U_{\rho}$ a solution of (19),

$$
\begin{aligned}
(B)= & -\int_{\frac{R}{2}}^{R}\left(-U_{\rho}^{\prime \prime}+\omega U_{\rho}\right) V_{\rho} d r-\frac{1}{4}\left(\int_{\frac{R}{2}}^{R} U_{\rho}^{2} d r\right)^{2} \int_{\frac{R}{2}}^{R} U_{\rho} V_{\rho} d r+\int_{\frac{R}{2}}^{R} U_{\rho}^{p} V_{\rho} d r \\
& -V_{\rho}(R) U_{\rho}^{\prime}(R)+o\left(e^{-\sqrt{k_{2}} \frac{R}{4}}\right) \\
= & \frac{1}{4}\left(\int_{-\infty}^{+\infty} U^{2} d r\right)^{2} \int_{\frac{R}{2}}^{R} U_{\rho} V_{\rho} d r-\frac{1}{4}\left(\int_{\frac{R}{2}}^{R} U_{\rho}^{2} d r\right)^{2} \int_{\frac{R}{2}}^{R} U_{\rho} V_{\rho} d r \\
& +\sqrt{k_{2}} e^{-2 \sqrt{k_{2}}(R-\rho)}+o\left(e^{-2 \sqrt{k_{2}}(R-\rho)}\right) \\
= & \sqrt{k_{2}} e^{-2 \sqrt{k_{2}}(R-\rho)}+o\left(e^{-2 \sqrt{k_{2}}(R-\rho)}\right) .
\end{aligned}
$$

Moreover, since $V_{\rho}$ is a solution of (20),

$$
\begin{aligned}
(C)= & \frac{1}{2} \int_{\frac{R}{2}}^{R}\left(-V_{\rho}^{\prime \prime}+\omega V_{\rho}\right) V_{\rho} d r+\frac{1}{8}\left(\int_{\frac{R}{2}}^{R} U_{\rho}^{2} d r\right)^{2} \int_{\frac{R}{2}}^{R} V_{\rho}^{2} d r+\frac{1}{2} V_{\rho}(R) V_{\rho}^{\prime}(R) \\
& +o\left(e^{-\sqrt{k_{2}} \frac{R}{4}}\right) \\
= & -\frac{1}{8}\left(\int_{-\infty}^{+\infty} U^{2} d r\right)^{2} \int_{\frac{R}{2}}^{R} V_{\rho}^{2} d r+\frac{1}{8}\left(\int_{\frac{R}{2}}^{R} U_{\rho}^{2} d r\right)^{2} \int_{\frac{R}{2}}^{R} V_{\rho}^{2} d r \\
& +\frac{1}{2} \sqrt{k_{2}} e^{-2 \sqrt{k_{2}}(R-\rho)}+o\left(e^{-2 \sqrt{k_{2}}(R-\rho)}\right) \\
= & \frac{1}{2} \sqrt{k_{2}} e^{-2 \sqrt{k_{2}}(R-\rho)}+o\left(e^{-2 \sqrt{k_{2}}(R-\rho)}\right) .
\end{aligned}
$$

Finally, since

$$
\left|\left(U_{\rho}-V_{\rho}\right)^{p+1}-U_{\rho}^{p+1}+(p+1) U_{\rho}^{p} V_{\rho}\right| \leqslant \frac{p(p+1)}{2} U_{\rho}^{p-1} V_{\rho}^{2},
$$

we have

$$
(D)=o\left(e^{-2 \sqrt{k_{2}}(R-\rho)}\right) .
$$

Then the conclusion follows.

The proof of Theorem 1.1 is completed with the following proposition. 
Proposition 4.2. Let us define $\Phi: \mathcal{I}_{R} \rightarrow \mathbb{R}$ as $\Phi(\rho)=I_{R}\left(z_{\rho}+w_{\rho}\right)$. Then,

$$
\Phi(\rho)=2 \pi J(U) \rho+2 \pi \sqrt{k_{2}} \rho e^{-2 \sqrt{k_{2}}(R-\rho)}+o\left(\rho e^{-2 \sqrt{k_{2}}(R-\rho)}\right) .
$$

Moreover, $\Phi$ attains a global minimum in the interior of $\mathcal{I}_{R}$ for $R$ sufficiently large.

Proof. First, we use Taylor expansion to get, for some $\xi_{\rho} \in(0,1)$,

$$
\begin{aligned}
I_{R}\left(z_{\rho}+w_{\rho}\right) & =I_{R}\left(z_{\rho}\right)+I_{R}^{\prime}\left(z_{\rho}\right)\left[w_{\rho}\right]+\frac{1}{2} I_{R}^{\prime \prime}\left(z_{\rho}+\xi_{\rho} w_{\rho}\right)\left[w_{\rho}, w_{\rho}\right] \\
& =I_{R}\left(z_{\rho}\right)+I_{R}^{\prime}\left(z_{\rho}\right)\left[w_{\rho}\right]+\frac{1}{2} I_{R}^{\prime \prime}\left(z_{\rho}\right)\left[w_{\rho}, w_{\rho}\right]+\frac{1}{2}\left(I_{R}^{\prime \prime}\left(z_{\rho}+\xi_{\rho} w_{\rho}\right)-I_{R}^{\prime \prime}\left(z_{\rho}\right)\right)\left[w_{\rho}, w_{\rho}\right] .
\end{aligned}
$$

By using Lemmas 3.1, 3.2, 3.3, we obtain that:

$$
I_{R}\left(z_{\rho}+w_{\rho}\right)=I_{R}\left(z_{\rho}\right)+O\left(\left\|w_{\rho}\right\|^{2}\right) .
$$

Proposition 3.4 and (21) imply that the error $\left\|w_{\rho}\right\|^{2}$ is negligible, so that

$$
\Phi(\rho)=2 \pi J(U) \rho+2 \pi \sqrt{k_{2}} \rho e^{-2 \sqrt{k_{2}}(R-\rho)}+o\left(\rho e^{-2 \sqrt{k_{2}}(R-\rho)}\right) .
$$

For the sake of brevity, we set

$$
\begin{aligned}
& \rho_{\alpha}=R-\frac{\alpha}{2 \sqrt{k_{2}}} \log R, \\
& \rho_{1}=R-\frac{1}{2 \sqrt{k_{2}}} \log R, \\
& \rho_{\beta}=R-\frac{\beta}{2 \sqrt{k_{2}}} \log R .
\end{aligned}
$$

We will prove that $\Phi$ has a minimum in the interior of $\mathcal{I}_{R}$, showing that $\Phi\left(\rho_{\beta}\right)$ $\Phi\left(\rho_{1}\right) \rightarrow+\infty$ and $\Phi\left(\rho_{\alpha}\right)-\Phi\left(\rho_{1}\right) \rightarrow+\infty$, as $R \rightarrow+\infty$. Indeed

$$
\begin{aligned}
& \Phi\left(\rho_{\beta}\right)-\Phi\left(\rho_{1}\right)=\frac{\pi(1-\beta) J(U)}{\sqrt{k_{2}}} \log R+O(1), \\
& \Phi\left(\rho_{\alpha}\right)-\Phi\left(\rho_{1}\right)=2 \pi \sqrt{k_{2}} R^{1-\alpha}+o\left(R^{1-\alpha}\right) .
\end{aligned}
$$

Remark 4.3. The solution that we have found corresponds to a minimum of the reduced functional, and recall that $w_{k_{2}}$ is also a minimizer for the limit functional J. Therefore, it is reasonable to think that our solution corresponds to a local minimum of $I_{R}$.

Moreover, its energy diverges negatively as $R \rightarrow+\infty$, so it could be the global minimizer for $I_{R}$. Indeed, by the arguments in [14], the global minimizer of $I_{R}$ must contain a term like $U_{\rho}(r)$, for some $\rho \sim R$. However, it is not clear if the global minimizer could also have a "localized part".

\section{REFERENCES}

[1] A. Ambrosetti, E. Colorado and D. Ruiz, Multi-bump solitons to linearly coupled systems of nonlinear Schrödinger equations, Calc. Var. Partial Differential Equations 30 (2007), 85-112.

[2] A. Ambrosetti and A. Malchiodi, Perturbation methods and semilinear elliptic problems on $\mathbb{R}^{N}$, Progress in Mathematics, 240. Birkhäuser Verlag, Basel, 2006.

[3] A. Ambrosetti, A. Malchiodi and W.-M. Ni, Singularly perturbed elliptic equations with symmetry: existence of solutions concentrating on spheres. II, Indiana Univ. Math. J. 53 (2004), 297-329.

[4] L. Bergé, A. de Bouard and J. C. Saut, Blowing up time-dependent solutions of the planar ChernSimons gauged nonlinear Schrödinger equation, Nonlinearity 8 (1995), 235-253.

[5] J. Byeon, H. Huh, and J. Seok, Standing waves of nonlinear Schrödinger equations with the gauge field, J. Funct. Anal. 263 (2012), no. 6, 1575-1608.

[6] C. Hagen, A new gauge theory without an elementary photon, Ann. of Phys. 157 (1984), 342-359.

[7] C. Hagen, Rotational anomalies without anyons, Phys. Review D 31 (1985), 2135-2136.

[8] H. Huh, Blow-up solutions of the Chern-Simons-Schrödinger equations, Nonlinearity 22 (2009) 967-974. 
[9] H. Huh, Standing waves of the Schrödinger equation coupled with the Chern-Simons gauge field. J. Math. Phys. 53 (2012), no. 6, 063702, 8 pp.

[10] H. Huh, Energy Solution to the Chern-Simons-Schrödinger Equations, Abstract and Applied Analysis Volume 2013, Article ID 590653, 7 pages.

[11] R. Jackiw and S.-Y. Pi, Soliton solutions to the gauged nonlinear Schrödinger Equations, Phys. Review Letters 64 (1990), 2969-2972.

[12] R. Jackiw and S.-Y. Pi, Classical and quantal nonrelativistic Chern-Simons theory, Phys. Rev. D 42 (1990) 3500-3513.

[13] R. Jackiw and S.-Y. Pi, Self-dual Chern-Simons solitons, Progr. Theoret. Phys. Suppl. 107 (1992) $1-40$.

[14] A. Pomponio and D. Ruiz, A Variational Analysis of a Gauged Nonlinear Schrödinger Equation, preprint arXiv:1306.2051.

[15] G. Tarantello, Self-Dual Gauge Field Vortices: An Analytical Approach, PNLDE 72, Birkhäuser Boston, Inc., Boston, MA, 2007.

${ }^{1}$ Dipartimento di Meccanica, Matematica e Management, Politecnico di Bari, Via E. ORABONA 4, 70125 BARI, ITALY.

${ }^{2}$ DPto. AnÁlisis Matemático, Granada, 18071 Spain.

E-mail address: a.pomponioepoliba.it, daruiz@ugr.es 